\title{
Action of the Loop Group on the Self Dual Yang-Mills Equation
}

\author{
Louis Crane* \\ Department of Mathematics, University of Chicago, Chicago, Illinois USA
}

\begin{abstract}
Recently Kac-Moody symmetry has played an important role in mathematical physics. Dolan and Chau, Ge and Wu discovered an infinitesimal action of the Kac-Moody Lie algebra on the space of solutions of SDYM. We have discovered an action of the loop group on the space of generalized solutions of SDYM, which exponentiates the Kac-Moody action. The group acts by adding a special type of source onto the solution. The action is a geometric construction using the twistor picture.
\end{abstract}

\section{Table of Contents}

Chapter

I. Introduction . . . . . . . . . . . . . . . . . . . 392

II. Background and Notation . . . . . . . . . . . . . 393

A. The Self Dual Yang-Mills Equation . . . . . . . . . 393

B. Kac-Moody Lie Algebras: Loop Groups . . . . . . . . 393

C. The Twistor Correspondence . . . . . . . . . . . . 395

D. Holomorphic Bundles, Birkhoff Decompositions, and RiemannHilbert Problems . . . . . . . . . . . . . . . . 396

III. The Infinitesimal Construction of Chau, Ge Wu, and Sinha. . . 397

A. The Infinitesimal Construction of Chau, $\mathrm{Ge} \mathrm{Wu}$, and Sinha . 397

B. Twistor Interpretation of the Infinitesimal Construction 399

IV. The Group Action Twistor Reformulation of the Problem . . . 399

A. Triviality and Unitarity Conditions . . . . . . . . . . 401

B. The Loop Group Action as a Riemann-Hilbert Problem . . 404

C. The Group Action in the G Formulation . . . . . . . . 404

D. The Larger Group . . . . . . . . . . . . . . . . 408

V. Specific Computations . . . . . . . . . . . . . . . . . . . 408

VI. Behavior at Infinity. . . . . . . . . . . . . . . . . 411

References . . . . . . . . . . . . . . . . . . . . 413

* Present address: School of Mathematics, Institute for Advanced Study, Princeton, NJ 08540, USA 


\section{Chapter I. Introduction}

Kac-Moody Lie algebras appear in mathematics in the theory of completely integrable ordinary differential equations and systems of partial differential equations in two variables [1]. The representation theory of Kac-Moody Lie algebras has played an important role in modern quantum field theory [2].

Consequently, it was considered very interesting when L. L. Chau et al. [3] and L. Dolan [4] discovered a representation of the Kac-Moody Lie algebra for $S U(N)$ on the space of solutions to the self dual Yang-Mills equation (SDYME) in four Euclidean dimensions. Chau and Dolan left open the problem of finding a group action corresponding to the (infinitesimal) action of the Kac-Moody algebra, and of providing a geometric interpretation. In addition, they did not specify which topological completion of the algebra acted. The normalization which they used to obtain a unique action of the algebra cannot actually be carried out, as there is a holomorphic obstruction.

In this paper we provide a reformulation of the situation in which the action of the algebra is easily understood by using the twistor program, as adapted to the Euclidean regime by Atiyah and Ward. Solutions of SDYME are coded into holomorphic bundles over $\mathrm{CP}^{3}$ [5], which are described by a transition function $\mathrm{G}$. The holomorphic bundle is regarded as a scattering problem, and its essential data is analogous to an associated linear problem, or "Lax pair." Elements of the loop group act by left and right multiplication on the transition functions, and the KacMoody algebra is identified as the Lie algebra of the loop group. The action of the loop group becomes simple and natural, and its effect can be analyzed by computation. The action of the algebra is clarified as a by-product.

The natural space for the loop group action is larger than the equivalence classes of solutions to SDYME. We show this by calculating the effect on 1-instanton solutions. The Kac-Moody action does not complete to $R^{4} \cup\{\infty\}=S^{4}$, and the loop group adds "sources" of a special type. It is also possible to define the action of a larger group; namely the holomorphic twistor matrix functions in a neighborhood of $R^{4} \times S^{1} \subset C P^{3}$. We conjecture that instanton adding transformations can be constructed from the larger group.

Our results are thus both positive and negative. We have a simple twistor picture of the Kac-Moody algebra action, which does not preserve finite energy conditions. The group action does not preserve the reality condition which keeps the solution of SDYME in $S U(N)$. On the other hand, our larger class of "solutions with sources" may have intrinsic interest; and our approach unifies treatment of reality conditions corresponding to the real forms $S U(N-M, M)$ of $S L(N, C)$.

The plan of the paper is as follows: In Chap. II we introduce notation and develop the necessary background material: the SDYME, Kac-Moody algebras and their actions, loop groups, the twistor correspondence, holomorphic bundles, Birkhoff decompositions, and Riemann-Hilbert problems. In Chap. III we review the construction of Chau et al. and reinterpret it in the twistor picture. In Chap. IV we propose a group action via solutions of Riemann-Hilbert problems. We then note that this action can be greatly simplified by reformulating the problem. We state and prove our main theorem in this section. We also demonstrate the existence 
of a larger group. In Chap. V we investigate the action of the loop group on the zero solution, and on the one-instanton solutions. In order to do this, we rewrite the oneinstanton solution in our formalism, where its simplicity is remarkable. We show the appearance of sources, under the action of special elements of the loop group. In Chap. VI we investigate the behavior of the group and algebra actions at infinity.

\section{Chapter II. Background and Notation}

\section{A. The Self Dual Yang-Mills Equation}

Gauge theories are at the heart of contemporary field theory. A gauge theory is defined by specifying dynamics for a unitary connection on a complex vector bundle over a Lorentzian manifold $M$. The Lagrangian used to specify the dynamics is the Yang-Mills Lagrangian

$$
L_{Y M}=\int d x\left|F_{\mu v} \cdot F^{\mu v}\right| \quad \text { (Einstein convention), }
$$

where $F_{\mu \nu}=\partial_{\mu} A_{\nu}-\partial_{\nu} A_{\mu}+\left[A_{\mu}, A_{\nu}\right]$ is the curvature of the connection $A$.

In any frame for the vector bundle, the connection is specified by a Lie algebra valued 1-form $A_{\mu}$, i.e., a section of $T^{*} \otimes s u(N) . F_{\mu v}$ is then a Lie algebra valued 2form. The dot in the definition of $L_{Y M}$ denotes the usual bi-invariant trace inner product on the Lie algebra $s u(N)$. The Euler Lagrange equation associated with $L_{Y M}$, $D_{\mu} F^{\mu v}=0$ (where $D_{\mu}=\partial_{\mu}+\left[A_{\mu},{ }^{*}\right]$ is the covariant derivative associated with $A$ ) is called the Yang-Mills equation. (for further discussion, see ref. 5)

The situation of most natural interest in physics is the Yang-Mills equation over a 4-dimensional Lorentzian space-time. However, in the Euclidean path integral approach, Yang-Mills equations over Riemannian 4-manifolds are also important.

Over a Riemannian 4-manifold $(M, g)$, there are two simpler systems of eauations $F_{\mu \nu}= \pm{ }^{*} F_{\mu \nu}$, where $*$ denotes Hodge duality, which in 4 dimensions takes 2-forms to 2-forms. These two systems are called the self dual and anti-self dual Yang-Mills equations. Since they are interchanged by a reversal of orientation, I refer to either as SDYME.

Any solution to SDYME is automatically a solution to the Yang-Mills equations. It is a conjectured that all global solutions to Yang-Mills on $S^{4}$ are solutions to SDYME. Solutions on $S^{4}$ to SDYME are called instantons. For $S U(2)$ bundles they are completely classified $[5,6]$.

The SDYME is conformally invariant. Hence solutions on $R^{4}$ and $S^{4}-\{\infty\}$ are equivalent. A solution of $R^{4}$ extends across $\infty$ on $S^{4}$ iff its curvature is squareintegrable [7].

To summarize: The subject matter of this paper is the solutions of the SDYME for $S U(N)$ bundles over $S^{4}, R^{4}$ or an open subset, with the standard metrics. Some generalization is possible [8] but we have not investigated it.

\section{B. Kac-Moody Lie Algebras: Loop Groups}

Let us start with any finite-dimensional Lie algebra with generators $T^{a}$ and structure 
constants $C_{a b c}$, i.e.,

$$
\left[T^{a}, T^{b}\right]=C_{a b c} T^{c}
$$

We can construct an infinite dimensional Lie algebra, which we shall refer to as a Kac-Moody algebra, with generators $T_{i}^{a}$, where $i \in Z$. The bracket is defined via

$$
\left[\begin{array}{cc}
T_{i}^{a} & T_{j}^{b}
\end{array}\right]=C_{a b c} T_{i+j}^{c}
$$

and is then extended by linearity to finite linear combinations of $T_{i}^{a}$, and by continuity to various completions.

Definition. An infinitesimal action of a Kac-Moody Lie algebra on a system of partial differential equations is a function which to each element of the algebra and each solution $s$ of the system assigns a solution of the linearized form of the system around $s$ and satisfies appropriate bracket relations.

Formally, the linearized form of the system may be derived by substituting $s+\varepsilon t$ into the system, and keeping only terms of the first order in the "infinitesimal parameter" $\varepsilon$. One likes to think of solutions of the linearized form as corresponding to 1-parameter families of solutions near $s$; but that is not always the case. One great merit of our approach to SDYME is that it deals with group actions, thus avoiding many mathematical complexities involving completions of algebras, differentiability properties, and exponentiation of infinitesimal actions. As we shall see, the group action we discover does not stay in the set of solutions to SDYME.

Infinitesimal actions of Kac-Moody algebras have been discovered on many important physical systems. These include dimensionally reduced general relativity [9], two-dimensional sigma models [10], and the sine-Gordon equation [11]. Since an infinite-parameter symmetry corresponds to an infinite family of conserved quantities, these actions have a deep relationship to integrable systems, and soliton behavior, which is not fully understood.

The systems with Kac-Moody actions seem to form a hierarchy. Solutions of the sine-Gordon equation form backbones for solutions of sigma models in two dimensions [12]. Dimensionally reduced gravity reduces to the Ernst equation [13], which can be thought of as a more complex two-dimensional sigma model $[29,30,14]$. The equation for axisymmetric monopoles, which is a specialization of SDYME, also reduces to the Ernst equation [13,15]. SDYME appears to be the most complex system in which the Kac-Moody action appears and to include the others as special cases. (As we shall see, it is natural to think of SDYME as a holomorphic problem in two complex dimensions. Kac-Moody symmetry seems to be a two-dimensional phenomenon.)

From our point of view, the Kac-Moody algebras appear as the Lie algebras of loop groups.

Definition. A loop group is the set of analytic maps of the circle into a Lie group

$$
\Omega G=C^{\omega}\left(S^{1}, G\right),
$$

with pointwise multiplication. 
An element in the tangent space to $\Omega G$ corresponds to a map from $S^{\prime}$ to the Lie algebra of $G$. Expanding such a map in Fourier coefficients gives the generators described above for a Kac-Moody algebra, plus a natural completion.

\section{The Twistor Correspondence}

Although Penrose developed the twistor program to understand fields in Minkowski space, it actually works equally elegantly in the Euclidean signature [6].

The Euclidean twistor approach begins with the observation that complex projective 3 -space fibres over the 4 -sphere with fibre the 2 sphere.

$$
S^{2} \rightarrow \underset{S^{4}}{\stackrel{C}{\downarrow} P F}
$$

$P F$ is the twistor fibration.

Let us describe $P F$. A point in $C P^{3}$ can be denoted $\left(\pi_{1}: \pi_{2}: \eta_{1}: \eta_{2}\right)$ as an equivalence class of 4-tuples of complex numbers, not all zero. Let us pick coordinates $\left(X^{1}, X^{2}, X^{3}, X^{4}\right)$ on $R^{4}=S^{4}-\{\infty\}$ : To a point in $R^{4}$ we associate a matrix

$$
\tilde{x}=\left(\begin{array}{rr}
x_{1}+i x_{2} & x_{3}+i x_{4} \\
-x_{3}+i x_{4} & x_{1}-i x_{2}
\end{array}\right)
$$

The solutions to the equation

$$
\left(\begin{array}{l}
\pi_{1} \\
\pi_{2}
\end{array}\right)=\tilde{x}\left(\begin{array}{l}
\eta_{1} \\
\eta_{2}
\end{array}\right)
$$

form a complex projective line in $C P^{3}$. Thus to each point in $R^{4}$ there corresponds a $C P^{1} \sim S^{2} \subset C P^{3}$. Every point in $C P^{3}$ lies on exactly one such line, which defines where $P F$ sends it; unless $\eta_{1}=\eta_{2}=0$, in which case $P F$ sends it to $\infty \in S^{4}$.

The inverse image of any point of $R^{4}$ under $P F$ can be parametrized by a complex parameter $\lambda=\eta_{2} / \eta_{1}$, which is holomorphic on $C P^{3}$.

The holomorphic structure of $C P^{3}$ is naturally described by four coordinate patches; one where each of the four projective coordinates is nonzero. If we delete the line over $\infty$, where $\eta_{1}=\eta_{2}=0$; then two patches suffice: $\eta_{1} \neq 0$, corresponding to $\lambda \neq \infty$, and $\eta_{2} \neq 0$, corresponding to $\lambda \neq 0$. We can introduce holomorphic coordinates.

$$
\left\{\lambda, \frac{\pi_{1}}{\eta_{1}}=w_{1}, \frac{\pi_{2}}{\eta_{2}}=w_{2}\right\} \text { and }\left\{\lambda^{-1}, \frac{w_{1}}{\lambda}, \frac{w_{2}}{\lambda}\right\} .
$$

If we introduce complex coordinates on $R^{4} y=\left(x_{1}+i x_{2}\right), z=\left(x_{3}-i x_{4}\right)$; we discover from (2) that $w_{1}=y+\lambda \bar{z}, w_{2}=\lambda \bar{y}-z$.

Thus, in using the twistor formulation over $R^{4}$, only two coordinate patches are needed. It is to be emphasized that $C P^{3}-C P_{\infty}^{1}$ is topologically a product, but that its holomorphic structure depends on $w_{1}$ and $w_{2}$ rather than $y$ and $z$, and is not a product. Also, the topological structure of $C P^{3}$ as a whole is not a product, $\lambda$ is not defined on $P F^{-1}(\infty)$, and our two coordinate patches do not extend there. The "half twist at infinity" is very important in the flavor of this theory. 
The point of introducing the twistor picture is that solutions of SDYME take on an elegant form, which is easy to analyze.

A bundle with a (unitary) connection over $S^{4}, R^{4}$, or any open subset can be pulled back along $P F$ to (an open subset of) $C P^{3}$. SDYME is just the right condition for the connection to "define a holomorphic structure" on the lifted bundle $[5,8]$.

Any connection on a complex bundle over a complex manifold can be used to lift the $\bar{\partial}$ operators from the manifold to the bundle. It is demonstrated in [6] that an integrability condition suffices to define a holomorphic structure on the bundle, and that that integrability conditon is exactly SDYME.

In coordinates, a section $s$ of the lifted bundle is holomorphic if

$$
\partial_{\bar{\lambda}} S=0, \quad \partial_{\bar{w}_{1}} S=\left(\lambda A_{y}-A_{\bar{z}}\right) s, \quad \partial_{\bar{w}_{2}} S=\left(\lambda A_{Z}+A_{\bar{y}}\right) s .
$$

We quote verbatim Theorem 5.2 from [8]: "Let $E$ be a hermitian vector bundle with self dual connection over a self dual manifold $X$, and let $F=p^{*} E$ be the pulled back bundle on $P\left(V_{-}\right)$. Then (1) $F$ is holomorphic on $P\left(V_{-}\right),(2) F$ is holomorphically trivial on each fibre, (3) there is a holomorphic isomorphism $\sigma: \tau^{*} \bar{F} \rightarrow F^{*}$, where $\tau: P\left(V_{-}\right) \rightarrow P\left(V_{-}\right)$is the real structure, such that $\sigma$ induces a positive definite hermitian structure on the space of holomorphic sections of $f$ on each fiber. Conversely, every such bundle on $P\left(V_{-}\right)$is the pullback of a bundle $E$ with self dual connection on $X$."

We do not need the generality of 5.2. For us, $P\left(V_{-}\right)=C P^{3}$ and $\tau$ sends $\lambda$ to $-1 / \bar{\lambda}$. Thus, we have a 1-1 correspondence between gauge equivalence classes of solutions to SDYME and holomorphic equivalence classes of holomorphic bundles over (suitable open subsets of) $C P^{3}$ satisfying (a) holomorphic triviality on fibres of $P F$, and (b) symmetry under $\lambda \rightarrow-1 / \bar{\lambda}$.

\section{Holomorphic Bundles, Birkhoff Decompositions, and Riemann-Hilbert Problems}

It should now be clear why we are interested in the theory of holomorphic bundles. Let us review those facts from the theory which we shall need [16].

a) A holomorphic bundle over a complex manifold is a bundle all of whose transition functions can be chosen to be holomorphic functions of the base.

$\beta$ ) (Grauert) Any holomorphic bundle over $C^{n}$ is trivial.

) (Grothendieck) Any holomorphic bundle over $C P^{1}$ is a direct sum of line bundles. Line bundles over $C P^{1}$ are classified by an integer (the first Chern class). Holomorphically, the set of integers in the decomposition of a vector bundle is invariant up to permutation. Topologically, however, only their sum is invariant. Hence the holomorphic structure of a bundle over $C P^{1}$ can change discontinuously under perturbation.

$\delta$ ) (Serre) Any holomorphic bundle over $C P^{n}$ has an algebraic structure (i.e., can be given a set of transition functions which are rational in the coordinates).

It follows from $\alpha$ and $\beta$ that a solution of SDYME over $R^{4}$ can be described by a single transition function $G(y, z, \lambda)$ between the two coordinate patches over $P F^{-1}\left(R^{4}\right) . G$ must be holomorphic in $w_{1}, w_{2}, \lambda$ in some neighborhood of $|\lambda|=1$ (for example), but can in fact be chosen holomorphic for $\lambda \neq 0, \infty$. 
We shall need to understand the holomorphic decomposition of a vector bundle over $C P^{1}$ in terms of its transition function given in a neighborhood of $|\lambda|=1$. This is equivalent to understanding the Birkhoff decomposition [17] of $G$.

Definition. Any holomorphic nonsingular matrix function of a complex variable $G(\lambda)$ defined in a neighborhood of the unit circle admits a decomposition

$$
G=\phi_{0} \Lambda \phi_{\infty}
$$

where $\phi_{0}$ is holomorphic in the interior of the unit circle, $\phi_{\infty}$ is holomorphic in the exterior, including $\infty$, and $\Lambda$ is a diagonal matrix whose entries are integral powers of $\lambda$. Such a decomposition is called a Birkhoff decomposition.

The exponents in the entries of $\Lambda$ are the Chern classes of the line bundles which occur in the holomorphic decomposition of the bundle with $G$ as transition function.

Definition. If $\Lambda$ is the identity matrix, i.e., if $G=\phi_{0} \phi_{\infty}$ then the decomposition is called a solution to the Riemann-Hilbert problem.

This name is historically inaccurate, but ubiquitous in the literature. If a solution of the Riemann-Hilbert problem exists for a given $G$, it is unique up to a transformation

$$
\begin{gathered}
\phi_{0}=\phi_{0} M \\
\phi_{\infty}=M^{-1} \phi_{\infty},
\end{gathered}
$$

where $M$ is a constant matrix.

The case in which $\Lambda=I$, i.e., in which the Riemann-Hilbert problem has a solution, is in fact generic [18]. Unfortunately, Birkhoff decompositions are hard to compute.

\section{Chapter III. The Infinitesimal Construction of Chau, $\mathrm{Ge}, \mathrm{Wu}$, and Sinha}

\section{A. The Infinitesimal Construction of Chau, Ge, $W u$, and Sinha}

In a series of three papers [3], the above authors constructed some actions of the $S U(N)$ Kac-Moody Lie algebra on the space of solutions of SDYME. Their construction hinged on the existence of an associated linear problem to SDYME, which emphasizes the connection with the theory of the inverse scattering transform [19]. We shall see later that the associated linear problem is closely related to the twistor picture.

The infinitesimal construction uses Yang's $J$ formalism [20]. Yang's idea is to regard the base manifold $R^{4}$ as $C^{2}$, i.e., to choose complex coordinates $y, z$. He then observed that SDYME can be written

$$
\begin{aligned}
& F_{y z}=F_{\bar{y} \bar{z}}=0, \\
& F_{y \bar{y}}+F_{z \bar{z}}=0 .
\end{aligned}
$$

Equations (3.1a) and (3.1b) now state that the connection is flat in certain complex 2planes in complexified 4-space. This means that the connection is pure gauge along these planes, i.e., there exist nonsingular $N \times N$ matrix functions of $y, z, \bar{y}$, and $\bar{z}$ 
denoted $D$ and $\bar{D}$ such that

$$
\begin{array}{ll}
A_{y}=D^{-1} D_{y}, & A_{z}=D^{-1} D_{, z}, \\
A_{\bar{y}}=\bar{D}^{-1} \bar{D}_{\bar{y},}, A_{\bar{z}}=\bar{D}^{-1} \bar{D}_{\bar{z}} .
\end{array}
$$

We still have to include Eq. (3.1c). If we write $J=D \bar{D}^{-1}$, then (3.1c) becomes

$$
\left(J^{-1} J_{y_{y}}\right)_{\bar{y}}+\left(J^{-1} J,_{z}\right)_{\bar{z}}=0
$$

an equation reminiscent of the equations of motion for the chiral model. Thus we see that SDYME can be thought of as analogous to a chiral model in two complex dimensions.

The associated linear problem for SDYME which Chau et al. use is

$$
\partial_{\bar{z}} \chi=\lambda\left(\partial_{y}+J^{-1} J_{, y}\right) \chi, \quad-\partial_{\bar{y}} \chi=\lambda\left(\partial_{z}+J^{-1} J_{, z}\right) \chi,
$$

where $\chi(y, z, \lambda)$ is a complex nonsingular $N \times N$ matrix function.

Definition. An associated linear problem for a system of PDEs is a system of PDEs in the variables of the original system plus auxiliary variables, linear in the auxiliary variables which has solutions for the auxiliary variables iff the original variables solve the original system.

Fact. Equation (3.3) is an associated linear problem for (3.2c).

It is important to note that the solution of (3.3) for a $J$ which satisfies (3.2c) is problematic. If we require $\chi$ to be holomorphic in $\lambda$ it will not, generally speaking, admit solutions globally in $\lambda$. Also, there is a great deal of nonuniqueness in $\chi$. Multiplying $\chi$ by any holomorphic matrix function $A(\lambda, \lambda \bar{y}+z, \lambda z-\bar{y})$ on the right gives another solution for $\chi$. Chau et al. attempt to make a unique choice of $\chi$ by specifying $\chi(\infty)=I$. As we shall see from the twistor picture, there is an obstruction to defining $\chi$ on all of $R^{4}$ plus $\infty$. Certainly the formulas for $\chi$ associated to a 1instanton solution published in an earlier paper [21] diverge at $\infty$. The solutions in [21] have poles at various values for $\lambda$. Thus there is a great deal of ambiguity in the following construction.

The first family of infinitesimal transformations is now written

$$
J^{-1} \delta_{\alpha}^{a} J=\chi(\alpha) T^{a} \chi^{-1}(\alpha),
$$

where $T^{a}$ is a generator of $S U(N)$. I have slightly modified the original notation to emphasize that the transformation depends on a complex parameter $\alpha$. (I will not discuss the calculation which shows that (3.4) is an infinitesimal transformation for (3.2c), because I do not use infinitesimal methods in the sequel.)

When $\delta_{\alpha}^{a}$ is expanded in a Taylor series in $\alpha$, the generators of a Kac-Moody algebra result.

Unfortunately, these transformations do not (infinitesimally) preserve the unitarity of the connection. In order to overcome this defect Chau et al. proposed a second family of infinitesimal transformations

$$
\tilde{\delta}_{\alpha}^{a} J=-J \tilde{X}(\alpha) T^{a} \tilde{X}(\alpha)^{-1},
$$


where $\tilde{X}(\alpha)$ satisfies (3.3) for $\hat{\lambda}=1 / \alpha$. It is then observed that

$$
\Delta_{\alpha}^{a}=\delta_{\alpha}^{a}+\tilde{\delta}_{-\alpha}^{a}, \quad \tilde{\Delta}_{\alpha}^{a}=-i\left(\delta_{\alpha}^{a}-\tilde{\delta}_{-\alpha}^{a}\right)
$$

do preserve unitarity. We end up with an infinitesimal action of a real form of the complex Kac-Moody Lie algebra generated from $S L(N, C)$; which acts on $S U(N)$ solutions of SDYME.

It is this picture of two actions, one "left" and one "right," plus a combination which preserves unitarity, which needs explanation. In summary, the work of Chau et al. has left the following questions open.

1. Is there an efficient way to compute these transformations?

2. Can the ambiguity in their definition be avoided? (As we shall see, not completely.)

3. What closure of the algebra of finite combinations of Kac-Moody generators actually acts?

4. What is the group action? Does the group action take solutions of SDYME to solutions? If not, on what space does it act?

5. Do the transformations converge at $\infty$ ?

6. Are instanton solutions taken into one another?

7. What is the geometric interpretation of the transformation?

As we shall see, the twistor approach enables us to shed light on these questions.

\section{B. Twistor Interpretation of the Infinitesimal Construction}

Before going on to construct the group action, we need to clarify the relationship between the formalism in this section and the twistor approach.

The equations which $D$ and $\bar{D}$ satisfy define them as holomorphic cross sections of the lifted bundle over $C P^{3}$ at $\lambda=0$ and $\lambda=\infty$ (written in a lifted frame). It is not surprising that they are gauge dependent. $J$ is then the transition matrix between the frames at $\lambda=0$ and $\infty$, pushed down to $R^{4}$ via $P F$. It is clear why $J$ is gauge invariant.

$\chi(\lambda)$ has a similar interpretation. If we choose a holomorphic frame for the lifted bundle in some region which includes $\lambda=0$, then $\chi(\lambda)$ is the transition matrix between the frames at $\lambda$ and at $\lambda=0$, pushed down to $R^{4}$. We can see that in general there will not be solutions for $\chi$ which are global in $\lambda$ or on $S^{4}$.

An important step in solving this problem is translating between the geometric picture of holomorphic frames and the physicist's picture of matrix functions on spacetime with a complex parameter $\lambda$.

\section{Chapter IV. The Group Action, Twistor Reformulation of the Problem}

One of the great merits of the work of Chau et al. was drawing attention to the associated linear problem, Eq. (3.3). The twistor interpretation of $\chi$ suggests that the bundle on twistor space should be thought of as analogous to the scattering data for a problem solvable by the inverse scattering transform [19]. In such a problem, the action of the Bäcklund transformations is simpler on the scattering data than on the original function. This suggests looking for loop group actions on bundles over $C P^{3}$. 
In order to compute the action of the loop group on a holomorphic bundle, we need to find some functions which describe the holomorphic structure of a bundle which satisfies the conditions of Theorem 5.2 quoted above. We satisfy this by means of the following theorem.

Theorem 1. If $A$ is a connection in $R^{4}$ expressed in some gauge which satisfies SDYME, then there exist two nonsingular matrix functions $\psi_{i}(y, z, \lambda) i=0, \infty$, with $\psi_{0}$ defined for $\lambda \neq \infty$ and $\psi_{\infty}$ defined for $\lambda \neq 0$, each satisfying

$$
\begin{aligned}
\left(\lambda A_{y}-A_{\bar{z}}\right) \psi_{i} & =\left(-\lambda \partial_{y}+\partial_{\bar{z}}\right) \psi_{i}, \\
\left(\lambda A_{z}+A_{\bar{y}}\right) \psi_{i} & =-\left(\lambda \partial_{z}+\partial_{\bar{y}}\right) \psi_{i}, \\
\partial_{\bar{\lambda}} \psi_{i} & =0 .
\end{aligned}
$$

Conversely, if $A$ possesses any solutions for (4.1) then A must satisfy SDYME.

Proof. Grauerts theorem ( $\beta$ of Chap. II, $\S D$ ) assures us that any holomorphic bundle on $C^{3}$ is holomorphically trivial. Thus we can find holomorphic trivializations of the bundle corresponding to $A$ in each of the coordinate patches described in Chap. II, Sect. C. We denote the two frames as $\psi_{0}, \psi_{\infty}$. These two frames can also be written as nonsingular matrices in the frame in which we have written $A$, lifted from $R^{4}$ to $C P^{3}-C P^{1}(\infty)$. By abuse of notation, we also call these matrix functions $\psi_{\infty}$ and $\psi_{0}$. Now we recall the definition of the holomorphic structure on the lifted bundle, Eqs. (2.3). Clearly, this is equivalent to (4.1).

The converse follow from noting that the integrability condition for $(4.1 \mathrm{a}, \mathrm{b})$ is just SDYME. We note that Theorem 1 is equally valid in a connected region in $R^{4}$ whose pullback under $P F$ is Stein, since a suitable generalization of Grauerts theorem holds [22], namely that the classifications of holomorphic and smooth bundles are identical for Stein manifolds.

Since our $\psi_{0}$ and $\psi_{\infty}$ are transition matrices between two different types of frames, they have two types of transformation laws. Under a change of frame on $R^{4}$ they transform $\psi \rightarrow P^{-1} \psi$. This is associated with a change in $A$. Under a holomorphic change of frame on $C P^{3}$, they transform $\psi \rightarrow \psi M$. Note that a holomorphic matrix on $C P^{3}$ is one for which the derivative operators in (4.1) give zero.

It is interesting to note that Eqs. $(4.1 \mathrm{a}, \mathrm{b})$ were written down by the physicists Belavin and Zakharov [23] as equations for the scattering of fermions through a solution of SDYME. Once again, the twistor picture looks like scattering data.

Equations $(4.1 \mathrm{a}, \mathrm{b})$ are also an associated linear problem for SDYME, i.e., their consistency is equivalent to SDYME. This is equivalent to the key step in the proof of the theorem numbered 5.2 in the cited paper of Atiyah.

Our $\psi$ functions are closely related to $\chi$. In fact $\psi_{0}^{-1}(0) \psi_{i}(\lambda)$ will solve the equation for $\chi(\lambda)$. We have succeeded in writing an infinitesimal transformation law for $\psi(\lambda)$ which reproduces Eq. (3.4) if we substitute $J=\psi_{\infty}^{-1}(\infty) \psi_{0}(0)$ and $\chi=\psi_{0}^{-1}(0) \psi_{0}(\lambda)$ :

$$
\delta_{\alpha}^{a} \psi_{i}(\lambda)=\frac{-\lambda}{\lambda-\alpha} \psi_{i}(\lambda)+\frac{\alpha}{\lambda-\alpha} \psi_{0}(\alpha)+T^{a} \psi_{0}^{-1} \alpha_{i}(\lambda)
$$


Instead of describing the lifted bundle via $\psi_{i}$, it is possible to use the transition matrix between them: $G(y, z, \lambda)=\psi_{\infty}^{-1} \psi_{0}$. $G$ must be nonsingular and holomorphic as a matrix function on $C P^{3}$ (i.e., holomorphic in the variables $w_{1}, w_{2}, \lambda$ ).

In order to define a holomorphic bundle on $C P^{3}-C P^{1}(\infty)$, it is sufficient to give a transition function holomorphic in a neighborhood of $|\lambda|=1$ (or real-analytic on $|\lambda|=1$ ). However, Grauert's theorem implies that it is always possible to change frames so that $G$ is holomorphic for $\lambda \neq 0, \infty$.

The approach which seems to clarify the Kac-Moody symmetry is first to find actions of the loop group on $\psi_{\infty}$ and $\psi_{0}$, then to reformulate in terms of $G$. In the $G$ formulation, the action becomes simple and computable.

Before discussing the group action, it is necessary to learn how to recognize when the bundle described via $\psi_{i}$ or $G$ satisfies the two conditions of Theorem 5.2 of Atiyah et al:: holomorphic triviality on fibres of $P F$, and existence of a real structure (or unitarity).

\section{A. Triviality and Unitarity Conditions}

It is complicated and superfluous to attempt a complete formulation of holomorphic triviality on fibres in the $\psi$ formalism. We merely note that (4.1c) implies that the lifted frame from $R^{4}$ is holomorphic on the copy of $C P^{1}$ over any point, and hence that the bundle is holomorphically trivial on fibres of $P F$.

Theorem 2. If a holomorphic bundle over $P F^{-1}(D)$ (where $\left.D \subset R^{4}\right)$ is constructed via a transition matrix $G(y, z, \lambda)$ between trivial bundles over $\lambda \neq 0$ and $\lambda \neq \infty$, the constructed bundle is trivial over $P F^{-1}\left(y_{0}, z_{0}\right)$ iff the Birkhoff decomposition of $G\left(y_{0}, Z_{0}, \lambda\right)$ reduces to a Riemann-Hilbert problem.

Proof. If $G=\phi_{0}^{-1} \phi_{\infty}$, then $\phi_{0} \psi_{0}=\phi_{\infty} \psi_{\infty}$, yielding a global holomorphic frame on $P F^{-1}\left(x_{0}, y_{0}\right)$. The converse is similar.

Since Birkhoff decompositions are difficult to compute, this appears to be a weakness of the $G$-formalism. In fact, it is a strength. The loop group action does not, in general, preserve holomorphic triviality on fibres. The natural set on which it acts is in fact more general than the space of solutions to SDYME; we interpret it as the space of solutions with sources. This is most clearly seen in the $G$ formalism.

The unitarity condition is also relatively easy to write down in the $\psi$ formalism. It is most efficient to find the condition for unitarity in an arbitrary signature; i.e., the condition for $A$ to be in $s u(m, N-m)$, the Lie algebra preserving a Hermitian form with $m$ positive and $N-m$ negative terms. In a unitary frame over $R^{4}$, this is the condition

$$
A_{x_{i}}^{\dagger} M+M A_{x_{\imath}}=0, \text { for all } i,
$$

where $\uparrow$ denotes hermitian conjugation and $M$ is a diagonal matrix with $m$ copies of +1 and $N-m$ copies of -1 .

Equation (4.3) can be recast in complex coordinates as

$$
A_{\bar{y}}=-M A_{y}^{\dagger} M^{-1}, \quad A_{\bar{z}}=-M A_{z}^{\dagger} M^{-1} .
$$


We now use Eq. $(4.1 \mathrm{a}, \mathrm{b})$ to write

$$
\begin{aligned}
\lambda A_{y}-A_{\bar{z}} & =\left[\left(-\lambda \partial_{y}+\partial_{\bar{z}}\right) \psi_{0}(\lambda)\right] \psi_{0}^{-1}(\lambda)-\frac{1}{\lambda} A_{z}+A_{\bar{y}} \\
& =-\left[\left(-\frac{1}{\bar{\lambda}} \partial_{z}+\partial_{\bar{y}}\right) \psi_{\infty}\left(\frac{-1}{\bar{\lambda}}\right)\right] \psi_{\infty}^{-1}\left(\frac{-1}{\bar{\lambda}}\right)
\end{aligned}
$$

at any $\lambda$, where $\psi_{0}$ is defined, and at whose antipode, $-1 / \bar{\lambda}, \psi_{\infty}$ is defined.

Now (4.4) implies

or

$$
\lambda A_{y}-A_{\bar{z}}+M\left(\bar{\lambda} A_{\bar{y}}-A_{z}\right)^{\dagger} M^{-1}=0
$$

$$
\left[\left(-\lambda \partial_{y}+\partial_{\bar{z}}\right) \psi_{0}(\lambda)\right] \psi_{0}^{-1} \lambda+M\left(\left[\left(\partial_{z}-\bar{\lambda} \partial_{\bar{y}}\right) \psi_{\infty}\left(\frac{-1}{\bar{\lambda}}\right)\right] \psi_{\infty}^{-1}\left(\frac{-1}{\bar{\lambda}}\right)\right)^{\dagger} M^{-1}=0
$$

or

$$
\left(\partial_{1} \psi_{0}(\lambda)\right) \psi_{0}^{-1}(\lambda)+M \psi_{\infty}^{-\dagger}\left(\frac{-1}{\bar{\lambda}}\right) M^{-1} \partial_{1}\left(M \psi_{0}^{-1}\left(\frac{-1}{\bar{\lambda}}\right) M^{01}\right)=0
$$

where $\partial_{1}=\lambda \partial_{y}+\partial_{\bar{z}}=\partial_{\bar{w}_{1}}$ and $\psi^{-\dagger}=\left(\psi^{\dagger}\right)^{-1}=\left(\psi^{-1}\right)^{\dagger}$. Multiplying (4.5) on the left by $M \psi_{\infty}^{\dagger}(-1 / \bar{\lambda}) M^{-1}$ and on the right by $\psi_{0}(\lambda)$ we obtain

$$
\partial_{\bar{w}_{1}}\left[M \psi_{\infty}^{\dagger}\left(\frac{-1}{\bar{\lambda}}\right) M^{-1} \psi_{0}(\lambda)\right]=0,
$$

and similarly for $\partial_{\bar{w}_{2}}$. Holomorphy in $\lambda$ is obvious. This implies that $M \psi_{\infty}^{\dagger}(-1 / \bar{\lambda}) M^{-1} \psi_{0}(\lambda)$ is a holomorphic matrix function on $C P^{3}$. This implies that by a new choice of $\psi_{0}$ we can set:

$$
\psi_{0}(\lambda)=M \psi_{\infty}^{-\dagger}\left(\frac{-1}{\bar{\lambda}}\right)
$$

This proves:

Theorem 3. Let $A$ be a solution of SDYME such that $A \varepsilon s u(m, N-m)$, then $\psi_{0}$ and $\psi_{\infty}$ can be chosen to satisfy $\psi_{0}(\lambda)=M \psi_{\infty}^{-\dagger}(-1 / \bar{\lambda})$, where $M=\operatorname{diag}(+1 \cdots+1$, $-1 \cdots-1)$ with signature $(m, N-m)$.

If we attempt to translate (4.6) into the $G$ formalism, we obtain only

or

$$
G\left(\frac{-1}{\bar{\lambda}}\right)=\psi_{\infty}^{-1}\left(\frac{-1}{\bar{\lambda}}\right) \psi_{0}\left(\frac{-1}{\bar{\lambda}}\right)=\psi_{0}^{\dagger}(\lambda) M^{-1} M \psi_{\infty}^{-\dagger}(\lambda)
$$

$$
G\left(\frac{-1}{\bar{\lambda}}\right)=\bar{G}(\lambda) .
$$

Theorem 4. If $A$ is a solution to SDYME, unitary in any signature, then $G$ may be chosen to satisfy $G(-1 / \bar{\lambda})=G^{\dagger}(\lambda)$.

The natural condition on $G$ does not distinguish the signature of $M$.

Once again, an apparent weakness of the $G$ formalism is in fact a strength. The action of the loop group does not, in general, preserve the signature of the unitary group. In this it is reminiscent of the Bäcklund transformation described in [24]. 
We should also note that (4.7) plus holomorphic triviality on fibres implies that if the bundle corresponds to a solution of SDYME then the connection is unitary in some signature. Thus if

and

$$
G\left(\frac{-1}{\bar{\lambda}}\right)=G^{\dagger}(\lambda)
$$

$$
\psi_{\infty}(\lambda) G(\lambda)=\psi_{0}(\lambda)
$$

then

and

$$
\psi_{\infty}\left(\frac{-1}{\bar{\lambda}}\right) G\left(\frac{-1}{\bar{\lambda}}\right)=\psi_{0}\left(\frac{-1}{\bar{\lambda}}\right),
$$

$$
\psi_{0}^{-\dagger}\left(\frac{-1}{\bar{\lambda}}\right) G^{\dagger}\left(\frac{-1}{\bar{\lambda}}\right)=\psi_{\infty}^{-\dagger}\left(\frac{-1}{\bar{\lambda}}\right),
$$

but $G^{\dagger}(-1 / \bar{\lambda})=G(\lambda)$, so that $\psi_{0}^{-\dagger}(-1 / \bar{\lambda}), \psi_{\infty}^{-\dagger}(-1 / \bar{\lambda})$ solve the same RiemannHilbert problem as $\psi_{\infty}(\lambda), \psi_{0}(\lambda)$. This implies

$$
\begin{aligned}
& \psi_{0}^{-\dagger}\left(\frac{-1}{\bar{\lambda}}\right)=\mu \psi_{\infty}(\lambda), \\
& \psi_{\infty}^{-\dagger}\left(\frac{-1}{\bar{\lambda}}\right)=\mu \psi_{0}(\lambda),
\end{aligned}
$$

where $\mu$ is a matrix independent of $\lambda$. Now,

so

$$
\psi_{\infty}(\lambda)=\mu^{-\dagger} \psi_{0}^{-\dagger}\left(\frac{-1}{\bar{\lambda}}\right)
$$

i.e.

$$
\psi_{0}^{-\dagger}\left(\frac{-1}{\bar{\lambda}}\right)=\mu \mu^{-\dagger} \psi_{0}^{-\dagger}\left(\frac{-1}{\bar{\lambda}}\right) \text {, }
$$

$$
\mu=\bar{\mu}
$$

Since $\mu$ is Hermitian it is possible to choose, on any contractible domain in $R^{4}$, a matrix function $k$ such that $k^{\dagger} \mu k=M$, where $M$ is a diagonal matrix of \pm 1 . Using $k$ as a gauge transformation, we now can recover Eq. (4.6), and therefore a solution to SDYME on any contractible domain. Two such solutions are related on their overlaps by gauge transformations.

Since $\pi_{1}(S U(N-m, m)) \neq 0$, it is in general possible that more than one patch may be required. We formulate the situation as follows.

Theorem 5. Any nonsingular holomorphic $N \times N$ matrix function $G(\lambda, y, z)$ defined on $C P^{3}-C P_{\infty}^{1}$ in a neighborhood of $|\lambda|=1$ satisfying $G^{\dagger}(\lambda)=G(-1 / \bar{\lambda})$ corresponds to a gauge equivalence class of solutions of SDYME, unitary in some signature, on each connected component of the region in which it has a trivial Birkhoff decomposition on fibres of $P F$.

Proof. It is only necessary to note that the proof of Theorem 5.2 cited above works 
equally well for a nonpositive definite real form. (See [5], p. 45.) Of course, the signature cannot change discontinuously. It is possible to have different signatures in regions separated by hypersurfaces on which $G$ gives nontrivial bundles along fibres of $P F$.

\section{B. The Loop Group Action as a Riemann-Hilbert Problem}

Zakharov and Shabat [25] have proposed a general method for defining an action of the loop group on integrable systems. They also indicated that their method could be extended to the SDYME. I have made an adaptation of their method to the $\psi_{\infty, 0}$ picture. This yields two actions of the loop group, which I interpret as the "left" and "right" actions of Chap. III. A combination is found which looks plausible as the unitarity preserving action. At this point, I reformulate the problem.

After completing this work, I learned that Ueno and Nakamura [26] did a similar analysis, although in a different formulation, which I am unable to interpret geometrically.

In the Zakharov-Shabat technique, one chooses a real analytic map from the unit circle to $S L(N, C)$, which I denote $g(\lambda)$. Any such map will have a holomorphic extension to some neighborhood of $|\lambda|=1$. One then solves the Riemann-Hilbert problem:

$$
\psi_{i}(\lambda) g(\lambda) \psi_{i}^{-1}(\lambda)=\phi_{\infty}^{i} \phi_{0}^{(i)}, \quad i=0, \text { or } \infty,
$$

where $\phi_{\infty}\left(\phi_{0}\right)$ is holomorphic in a neighborhood of the exterior (interior) of the unit circle, including $\infty(0)$.

Since the Riemann-Hilbert problem can only be solved "generically," we have the problem that (4.8) may not have a solution for all values of $y$ and $z$. We postpone consideration of this and all similar technical problems until after reformulation.

The two choices of $i$ in (4.8) will give the left and right actions. I will consider only the case $i=\infty$, and drop the superscript from $\phi$. Equations (4.1a) and (4.1b) can be written

where

$$
\partial_{i} \psi_{\infty}=A_{i} \psi_{\infty}, \quad i=1,2,
$$

$$
\begin{aligned}
& \partial_{1}=-\lambda \partial_{y}+\partial_{\bar{z}}, \quad \partial_{2}=-\left(\lambda \partial_{z}+\partial_{\bar{y}}\right), \\
& A_{1}=\lambda A_{y}-A_{\bar{z}}, \quad A_{2}=\lambda A_{z}+A_{\bar{y} .}
\end{aligned}
$$

These allow us to compute derivatives of both sides of (4.8). We obtain

$$
\left(\partial_{i} \phi_{\infty}\right) \phi_{0}+\phi_{\infty}\left(\partial_{i} \phi_{0}\right)=A_{i} \psi_{\infty} g \psi_{\infty}^{-1}-\psi_{\infty} g \psi^{-1} A_{i}=A_{i} \phi_{\infty} \phi_{0}-\phi_{\infty} \phi_{0} A_{i} .
$$

Rearranging, we find

$$
\phi_{\infty}^{-1} A_{i} \phi_{\infty}-\phi_{\infty}^{-1} \partial_{i} \phi_{\infty}=\phi_{0} A_{i} \phi_{0}^{-1}+\left(\partial_{i} \phi_{0}\right) \phi_{0}^{-1}=A_{i}
$$

The form of (4.9) is very important. It states that two gauge transformations over open subsets of $C P^{3}$ have equal effects on $A_{i}$. Since $\phi$ is holomorphic for a neighborhood of $|\lambda| \leqq 1$, while $\phi_{\infty}$ is holomorphic for $|z|>1$, the expression can add no new poles to the structure of $A_{i}$; thus it must be linear in $\lambda$. This gives us new, transformed values for $A_{y}, A_{\bar{y}}, A_{z}, A_{\bar{z}}$. We can check that the transformed values for $A$ 
solve SDYME by using the fact that Eq. (4.1) are an associated linear problem for SDYME, i.e., that existence of solutions for $\psi$ in (4.1) implies that $A$ satisfies SDYME.

The fact that (4.9) has the form of a gauge transformation implies that if we define

$$
\psi_{\infty}^{\prime}=\phi_{\infty}^{-1} \psi_{\infty}=\phi_{0} \psi_{\infty} g^{-1}, \quad \psi_{0}^{\prime}=\phi_{0} \psi_{0}
$$

(because $\phi_{0} \phi_{\infty}=\psi_{\infty} g \psi_{\infty}^{-1}$ ). Then $\psi_{0}^{\prime}$ and $\psi_{\infty}^{\prime}$, together with the transformed $A$ from (4.9), solve Eq. (4.1). We have now proved

Theorem 6. In any region in which (4.8) can be solved, (4.9) and (4.10) define a new solution for SDYME. In general, that solution need not be unitary.

By an argument analogous to the one given in [27] for the chiral model (one writes an integral equation for the Riemann-Hilbert problem, and solves it infinitesimally in $g(\lambda)$ ). It is now easy to see that the infinitesimal form of (4.9) and (4.10) gives (4.2). Similarly, using (4.8) for $i=0$ will give (3.5), since $\tilde{\chi}$ could easily be chosen to come from $\psi_{0}$.

At this point in the analysis, it is not clear what combination of the two RiemannHilbert problems would preserve unitarity. Further work in this direction was made superfluous by a reformulation of the problem.

\section{The Group Action in the G Formulation}

If we attempt to find the transition function corresponding to the bundle associated with the new solution, we find

$$
G^{\prime}=\psi_{\infty}^{\prime-1} \psi_{0}^{\prime}=\left(g \psi_{\infty}^{-1} \phi_{0}^{-1}\right)\left(\phi_{0} \psi_{0}\right)=g G .
$$

Similarly, the transformation developed from (4.8) by using $i=0$ gives $G \rightarrow G g^{-1}$. We have demonstrated the following:

Theorem 7. The actions of the loop group defined by (4.9) and (4.10) act by left and right multiplication on the function $G$ in a neighborhood of $|\lambda|=1$. (If we use only analytic elements of the loop group, they always extend to a neighborhood of the unit circle.)

The combination of left and right actions which preserves unitarity is now easily determined. Recall from Theorems 4 and 5 that the condition for unitarity is

$$
G\left(\frac{-1}{\bar{\lambda}}\right)=G^{\dagger}(\lambda)
$$

This is clearly preserved by

$$
T g(G)=g(\lambda) G(\lambda) g^{\dagger}\left(\frac{-1}{\bar{\lambda}}\right) .
$$

Corollary. Let $G$ be a transition function satisfying $G(-1 / \lambda)=G^{\dagger}(\lambda)$ corresponding to a solution of SDYME which is unitary in some signature. In any connected region in which $T g(G)$ considered as a function of $\lambda$ only has trivial Birkhoff decomposition, $\mathrm{Tg}(G)$ corresponds to a gauge equivalence class of solutions of SDYME, unitary in 
some signature. The signature associated with $T g(G)$ may not be the same as the original one.

We see that the transformation on $G$ is very simple. We therefore wish to regard $G$ rather than $A$ as the fundamental data for this problem. Unfortunately, the correspondence between $A$ and $G$ is not simple. We summarize the facts about this correspondence in the following:

Proposition 1. (a) Gauge equivalent A's correspond to the same set of G's. (b) Two different G's which generate holomorphically equivalent bundles correspond to the same equivalence class of $A$ 's. (c) If $G$ restricted to a fibre of PF generates a holomorphically trivial bundle, then at the corresponding point in $R^{4}$ we have a value for $A$.

Definition. A fibre for $P F$ on which $G$ has a nontrivial Birkhoff decomposition (generates a holomorphically nontrivial bundle) is called a jumping line for $G$.

Proof. Although the above facts all follow from the powerful abstract theorems we have quoted, let us demonstrate them prosaically in terms of matrix functions. (a) A gauge transformation on $A$ multiplies both $\psi_{0}$ and $\psi_{\infty}$ on the left by a transformation matrix independent of $\lambda$

$$
\psi_{0} \rightarrow M \psi_{0}, \quad \psi_{\infty} \rightarrow M \psi_{\infty}
$$

We then see that $G \rightarrow\left(M \psi_{\infty}\right)^{-1} M \psi_{0}=G$.

(b) If $G_{1}$ and $G_{2}$ give rise to holomorphically equivalent bundles, then we can write

$$
G_{1}=H_{\infty} G_{2} H_{0}
$$

where $H_{0}\left(H_{\infty}\right)$ is holomorphic in a neighborhood of $|\lambda| \leqslant 1(\geqslant 1)$ in the Riemann sphere. If $G_{2}$ can be written $\psi_{\infty}^{-1} \psi_{0}$, where $\psi_{i}$ solve (4.1) for some value of $A$, then $G_{1}$ can be written $\left(\psi_{\infty} H_{\infty}\right)^{-1} \psi_{0} H_{0}$. Since multiplication on the right by a holomorphic matrix function of $C P^{3}$ does not change Eq. (4.1), we see that the bundles generated by $G_{1}$ and $G_{2}$ correspond to the same $A$.

(c) Finally, if $G$ generates a bundle which is holomorphically trivial on fibres of $P F$, we can choose $\psi_{i}$ to be holomorphic in $\lambda$. There can be no topological obstruction to doing this globally; since any $S L(n, c)$ bundle on any open subset of $R^{4}$ is trivial.

Now if we define

$$
A_{i}=\left(\partial_{i} \psi\right) \psi^{-1}
$$

(notation as in the proof of (4.11)) then we see that the values of $A_{i}$ calculated from $\psi_{0}$ and $\psi_{\infty}$ will match because $G$ is holomorphic. Hence we see that $A_{i}$ is holomorphic except at $\infty$, where it has a simple pole from the $\lambda$ in the definition of $\partial_{i}$. Decomposing $A_{i}$ into constant and linear terms in $\lambda$, we recover an $A$ on $R^{4}$.

The ambiguity in $G$ matches the ambiguity in $\chi$ which we pointed out in Chapter III. Hence it is implicit in the nature of the loop group action. To phrase matters differently, the ambiguity in the loop group action on $A$ is unavoidable, because it is precisely the ambiguity in $G$ for a given $A$. 
It is interesting to examine the ambiguity in $G$ more closely. If $G_{1}$ and $G_{2}$ satisfy the unitarity condition and define holomorphically equivalent bundles, then we can write $G_{1}(\lambda)=H_{1}(\lambda) G_{2}(\lambda) H_{1}^{\dagger}(-1 / \bar{\lambda})$, where $H_{1}$ is holomorphic in the exterior of $|\lambda|=1$ including $\infty$. Thus the ambiguity in $G$ is just the action of a transformation very similar to $T g$, except that $g(\lambda)$ is not constant in $y$ and $z$. This means that the ambiguity in $G$ is equivalent, in its effect on the loop group action, to choice of a conjugacy class of the loop group in a larger group. We discuss the larger group later.

For all the reasons cited we regard the action $T g$ on $G$ as the fundamental loop group action. We now have to make a choice as to which loop group to use. Since we want to act on holomorphic $G$ 's, we need to be able to extend $g(\lambda)$ holomorphically in a neighborhood of $|\lambda|=1$. This implies that we must consider real analytic loops in a complex group, i.e., $C^{\omega}\left(S^{1}, S L(N, C)\right)$ or $C^{\omega}\left(S^{1}, G L(N, C)\right)$. We choose to consider only the former, i.e., we require $\operatorname{det}(g(\lambda))=1$.

There are several reasons for this choice. If $\psi_{0}, \psi_{\infty}$ can be chosen to be algebraic, then their determinants must be constant in $\lambda^{\prime}$, since any nonconstant algebraic function has zeros. This implies $\operatorname{det} G=$ constant, so it seems natural to consider only $g$ 's which preserve that property. Also, the group $G L(N, C)$ is not simply connected, so $\Omega G L(N, C)$ is not connected.

There is an advantage to considering the action of a connected loop group; namely, it cannot change the topological invariant of the bundle over any fibre of $P F$. The sum of the Chern classes of the line bundles in its decomposition remains zero.

Motivated by Theorem 4, we make the following definitions:

Definition. A generalized solution to SDYME on an open subset $S$ of $R^{4}$ is a holomorphic $N \times N$ nonsingular matrix function $G$ defined on a neighborhood in $P F^{-1}(S)$ of $|\lambda|=1$ satisfying $G(\lambda)=G^{\dagger}(-1 / \bar{\lambda})$ which defines a topologically trivial bundle. The points of $S$ where $G$ does not define a bundle which is holomorphically trivial on fibres of $P F$ are called sources of $G$.

A generalized solution to SDYME has a natural geometric interpretation as a holomorphic bundle on $C P^{3}$ (or an open subset) which is topologically trivial on fibres of $P F$. A generalized solution with no sources is, by Theorem 4 , simply a solution of SDYME. I believe that there should be some field which describes the sources.

It is interesting to think of my generalized solutions as analogous to the KaluzaKlein description of the Dirac monopole [28]. There again, a "source" and a field are combined into a single geometric entity, namely an 5-dimensional solution to Einstein's equation.

We now state our main theorem.

Theorem 8. The formula $\operatorname{Tg}(\lambda)(G(\lambda, y, z))=g(\lambda) G(\lambda, y, z) g^{\dagger}(-1 / \bar{\lambda})$ defines an action of $\Omega S L(N, C)$ on the space of generalized solutions of SDYME. The action of any element which extends holomorphically across the outer half of $C P^{1}$ does not change the sources of $G$ or the solution of SDYME in the source free regions. The action of the tangent space to $\Omega S L(N, C)$ at I gives an action of the Kac-Moody algebra which preserves unitarity.

It is now easy to see what infinite-dimensional algebra acts naturally, namely the 
tangent space to $C^{\omega}\left(S^{1}, S L(N, C)\right)$, or the space of convergent series in the generators for the Kac-Moody algebra.

If we look back at the list of questions we asked in Sect. $A$ of Chap. III, we see that $1,2,3,4$, and 7 are now answered. An answer to 5 and 6 depends on calculation of examples, which we shall consider in later chapters.

\section{The Larger Group}

If we examine the group action in Eq. (4.12), we see that the requirement that $g(\lambda)$ not depend on $y, z$ is superfluous. We can equally well define an action for all holomorphic matrix functions of a neighborhood of $|\lambda|=1$ in twistor space which have determinant 1 . For instance, any matrix of polynomials in $\lambda^{-1}, \lambda, w_{1}$ and $w_{2}$ with constant determinant has an action. An interesting example is

$$
\left(\begin{array}{cc}
1+\frac{w_{1} w_{2}}{\lambda} & \frac{w_{2}^{2}}{\lambda} \\
\frac{-w_{1}^{2}}{\lambda} & 1-\frac{w_{1} w_{2}}{\lambda}
\end{array}\right)
$$

which, as we shall see in the next chapter, adds an instanton to the vacuum. We do not feel that we understand the larger group yet.

\section{Chapter V. Specific Computations}

One merit of the $G$-formulation is its computational simplicity. It is now possible to discover the phenomenology of the loop group action by multiplication of polynomial matrices. We have not exhausted the possibilities of this method, but what we have discovered is intriguing.

The two natural solutions of SDYME to let the loop group act on are the vacuum solution $\psi_{i}=G=I$, and the instanton solutions. In order to do anything with the instanton solutions it is necessary to transform them into the $\psi_{\infty, 0}$ and $G$ formalisms. We did this only for the simplest 1-instanton solution. As far as we know our result has not appeared elsewhere.

Belavin and Zakharov [23] found the following representation for the simplest 1 -instanton solution (the others can be found via conformal transformations),

$$
\psi_{B Z}=\frac{1}{\left(2 R^{2}+1\right)^{\frac{1}{2}}}\left[\begin{array}{cc}
R^{2}+1+\lambda \bar{y} \bar{z}+y z / \lambda & \lambda \bar{y}^{2}-z^{2} / \lambda \\
-\lambda \bar{z}^{2}+y^{2} / \lambda & R^{2}+1-\lambda \bar{y} \bar{z}-y z / \lambda
\end{array}\right]
$$

(the notation is due to [21]) where $R^{2}=y \bar{y}+z \bar{z}$. This expression has poles both at $\lambda=0$ and at $\lambda=\infty$. Consequently it is not a candidate either for $\psi_{0}$ or $\psi_{\infty}$. Geometrically, $\psi_{B Z}$ represents a meromorphic frame for the 1-instanton bundle on twistor space. To compensate, it has the advantage of a symmetry

$$
\psi_{B Z}\left(\frac{-1}{\bar{\lambda}}\right)=\psi_{B Z}^{\dagger}(\lambda)
$$

In order to find a candidate for $\psi_{0}$, it was necessary to find a holomorphic matrix 
function on $C P^{3}$ which cancelled the pole at $\lambda=0$ but introduced no other singularities, and preserved the determinant. Several weeks of agonizing trial and error produced the following miraculous formula:

$$
\begin{gathered}
\frac{1}{\sqrt{2 R^{2}+1}}\left[\begin{array}{cc}
R^{2}+1+\lambda \bar{y} \bar{z}+\frac{y z}{\lambda} & \lambda \bar{y}^{2}-\frac{z^{2}}{\lambda} \\
-\lambda \bar{z}^{2}+\frac{y^{2}}{\lambda} & R^{2}+1-\lambda \bar{y} \bar{z}-\frac{y z}{\lambda}
\end{array}\right]\left[\begin{array}{cc}
1+\frac{w_{1} w_{2}}{\lambda} & \frac{w_{2}^{2}}{\lambda} \\
\frac{-w_{1}^{2}}{\lambda} & 1-\frac{w_{1} w_{2}}{\lambda}
\end{array}\right] \\
=\frac{1}{\sqrt{2 R^{2}+1}}\left[\begin{array}{cc}
1+2 y \bar{y}+2 \lambda \bar{y} \bar{z} & 2 \bar{y}^{2} \lambda-2 \bar{y} z \\
-2 \lambda \bar{z}^{2}-2 \bar{z} y & 1+2 z \bar{z}-2 \bar{y} \bar{z} \lambda
\end{array}\right]=\psi_{0}^{(B Z) .}
\end{gathered}
$$

I do not know how to explain the simplicity of this result; close to $90 \%$ of the terms in the expansion cancelled. We can now choose $\psi_{\infty}^{(B Z)}(\lambda)=\psi_{0}^{-\dagger}(-1 / \bar{\lambda})^{(B Z)}$ and calculate $G_{B Z}=\psi_{\infty}^{-1}(\lambda) \psi_{0}(\lambda)$. We obtain the square of our original holomorphic matrix, which, since it is built up out of nilpotents, is very simple:

$$
\begin{aligned}
G_{(B Z)} & =\left[\begin{array}{cc}
1+\frac{2 w_{1} w_{2}}{\lambda} & \frac{2 w_{2}^{2}}{\lambda} \\
\frac{-2 w_{1}^{2}}{\lambda} & 1-2 \frac{w_{1} w_{2}}{\lambda}
\end{array}\right] \\
& =\left[\begin{array}{cc}
1+2 y \bar{y}-2 z \bar{z}+2 \lambda \bar{y} \bar{z}-\frac{2 y z}{\lambda} & -4 \bar{y} z+2 \bar{y}^{2} \lambda+\frac{2 z^{2}}{\lambda} \\
-4 y \bar{z}-\frac{2 y^{2}}{\lambda}-2 \lambda z^{2} & 1+2 z \bar{z}-2 y \bar{y}+\frac{2 y z}{\lambda}-2 \bar{y} \bar{z} \lambda
\end{array}\right] .
\end{aligned}
$$

We think the fact that the one-instanton solution can be expressed so simply in the $G$ formulation is remarkable.

One interesting fact about Eq. (5.2) is that at the origin it reduces to the identity. Thus, any calculation on the vacuum solution is simultaneously a calculation at one point of the 1-instanton solution. It is also important to notice that $G_{(B Z)}$ is the square of our original holomorphic matrix, which is carried into itself by $g(\lambda) \rightarrow g^{\dagger}(-1 / \lambda)$; thus if we define

then

$$
g_{B}(\lambda, y, z)=\left[\begin{array}{cc}
1+\frac{w_{1} w_{2}}{\lambda} & \frac{w_{2}^{2}}{\lambda} \\
\frac{-w_{1}^{2}}{\lambda} & 1-\frac{w_{1} w_{2}}{\lambda}
\end{array}\right],
$$

$$
G_{B Z}^{(\lambda, y, z)}=g_{B}(\lambda, y, z) g_{B}^{\dagger}\left(\frac{-1}{\lambda}, y, z\right) .
$$

Equation (5.3) has two interpretations. If we think of $g_{B}$ as a fixed element of the loop group for a fixed $(y, z)$, then it tells us that the loop group action can convert the identity into any fibre of $P F$ in the 1-instanton solution. This means that anything which can happen to the trivial solution can happen at any point of the 1-instanton 
solution and vice versa. The second interpretation is to consider $g_{B}$ as an element of the larger group we described in the last section. Equation (5.3) then shows that this particular group element transforms the vacuum into the 1 -instanton solution. It would be interesting to determine whether acting with $g_{B}$ on an instanton centered at another point gives a 2 -instanton solution. That could be ascertained by finding the Birkhoff decomposition of $g_{b}(\lambda, y, z) g_{b}^{2}\left(\lambda, y-y_{0}, z-z_{0}\right) g_{b}(\lambda, y, z)$; but time pressure has not permitted us to do the calculation. Nevertheless it seems plausible that some element in the larger group could be an instanton adding transformation.

Returning to our study of the loop group, we see that in order to test whether the loop group adds sources to the 1-instanton solutions we need only search for a loop group element which takes the identity to a matrix with a nontrivial Birkhoff decomposition.

By trial and error, we discover that if

then

$$
g(\lambda)=\frac{1}{\sqrt{2}}\left[\begin{array}{cc}
1 & \lambda \\
\frac{-1}{\lambda} & 1
\end{array}\right]
$$

$$
g(\lambda) g^{\dagger}\left(\frac{-1}{\bar{\lambda}}\right)=\left[\begin{array}{cc}
0 & \lambda \\
-\lambda^{-1} & 0
\end{array}\right],
$$

which clearly has Chern classes +1 and -1 in its decomposition. We have proven

Proposition 2. The action of elements of the loop group can add sources to the 1instanton solution.

By calculating the Birkhoff decompositions of various polynomial matrices, it would be possible to determine exactly where sources are added.

Similarly, it is easy to see that loop group elements can change the signature at points. We note that if

then

$$
g(\lambda)=\left[\begin{array}{cc}
\lambda & 0 \\
0 & \lambda^{-1}
\end{array}\right],
$$

$$
g(\lambda) g^{\dagger}\left(\frac{-1}{\bar{\lambda}}\right)=\left[\begin{array}{rr}
-1 & 0 \\
0 & -1
\end{array}\right] \text {. }
$$

Since we are restricting ourselves to $\operatorname{det} g=1$, it is not possible to end up in $S U(1,1)$. However, for $N \geqq 3$, it is possible to have regions with noncompact forms of the unitary group for the connection. Since the signature of a definite hermitian form cannot change discontinuously, any path in loop space from the identity to an element which changes the signature at some point must pass through a point which induces a source. Further computation could greatly clarify this picture.

In one special case, we have been able to use algebraic means to calculate the effect of a 1-parameter family of loop group elements on the 1-instanton $G_{B Z}$ of (5.2); and to find the Birkhoff decomposition and locate the jumping lines. The idea is to use

$$
G=\psi_{\infty}^{-1} \psi_{0}
$$


and

$$
\psi_{\infty}(\lambda)=\psi_{0}^{-\dagger}\left(\frac{-1}{\bar{\lambda}}\right)
$$

Thus if we write

$$
\widehat{G}(\lambda)=f_{\varepsilon}(\lambda) G(\lambda) f_{\varepsilon}^{\dagger}\left(\frac{-1}{\bar{\lambda}}\right),
$$

we can try to find a $U_{\varepsilon}(\lambda)$ such that:

$\alpha) \hat{\psi}_{0}(\lambda)=U_{\varepsilon}(\lambda) \psi_{0}(\lambda) f_{\varepsilon}^{\dagger}(\lambda)$ is holomorphic for $\lambda \neq \infty$ and

в) $U^{\dagger}\left(\frac{-1}{\bar{\lambda}}\right) U(\lambda)=m I$.

For $f_{\varepsilon}$ we choose

$$
\frac{1}{\sqrt{1-\varepsilon \bar{\varepsilon}}}\left[\begin{array}{cc}
1 & \frac{\varepsilon}{\lambda} \\
\bar{\varepsilon} \lambda & 1
\end{array}\right],
$$

and we guess $U_{\varepsilon}(\lambda)=\left(I+(\hat{\varepsilon} / \lambda) \hat{N}+\bar{\varepsilon} \lambda \hat{N}^{\dagger}\right)$, where $\hat{\varepsilon}$ is a function, not a constant like $\varepsilon$, and $\hat{N}=\psi_{0}(0)\left(\begin{array}{ll}0 & 1 \\ 0 & 0\end{array}\right) \psi_{0}^{-1}(0)$, in order to cancel the pole in $\bar{\psi}_{0}$ at 0 . We find that $(\alpha)$ demands

$$
\hat{\varepsilon}=\frac{\varepsilon}{1-\varepsilon \rho},
$$

where $\rho$ is the lower left-hand entry of $\psi_{0}^{-1}(0) \partial_{\lambda} \psi_{0}(0)$. Now we discover that

$$
U U^{T}=(1-\hat{\varepsilon} \hat{\varepsilon} k) I,
$$

where $k=\hat{N}^{\dagger} \hat{N}+\hat{N} \hat{N}^{\dagger}$, so $(\beta)$ is satisfied unless $1-\hat{\varepsilon} \bar{\varepsilon} k=0$.

For $1-\varepsilon \hat{\varepsilon} k<0, G$ satisfies the $(0,2)$ unitarity condition. It follows that when $1-\varepsilon \hat{\varepsilon} k=0$, we have jumping lines, since the signature cannot change smoothly. Substituting for $\psi_{0}$ our expression $\psi_{0}^{B Z}$, we obtain

$$
1-\hat{\hat{\varepsilon}} \overline{\hat{c}} k=1-\frac{\left|\varepsilon^{2}\right|}{\left|\sqrt{2 R^{2}+1}-2 \varepsilon \bar{y} \bar{z}\right|^{2}}\left[1+r y \bar{y}\left(R^{2}+1\right)\right]^{2} .
$$

Equation (5.4) implies that any ray not in the $y$-plane eventually hits a jumping line. $(0,0)$ is not a jumping line until $\varepsilon=1$. As $\varepsilon \rightarrow 0$, the hypersurface of jumping lines goes to $\infty$.

\section{Chapter VI. Behavior at Infinity}

The formalism we have adopted is not well adapted to studying the behavior of solutions to SDYME at the point $\infty$. The two coordinate patches we chose on $C P_{3}-C P_{\infty}^{1}$ do not extend there, neither do $\psi_{\infty}, \psi_{0}$, or $G$. We can attempt to understand how our loop group action looks at $\infty$ by finding a transformation on $C P^{3}$ which exchanges 0 and $\infty$ on $S^{4}$, then seeing how the transformation changes 
our loop group action. Thus, if we map

we find that

$$
\begin{aligned}
& \pi_{i} \rightarrow \eta_{i}, \\
& \eta_{i} \rightarrow \pi_{i},
\end{aligned}
$$

$$
\begin{aligned}
& y \rightarrow \frac{\bar{y}}{R^{2}} z \rightarrow \frac{-z}{R^{2}}, \\
& \lambda \rightarrow \frac{y+\bar{z} \lambda}{-z+\bar{y} \lambda} .
\end{aligned}
$$

Equations (6.1) tell us that in coordinates centered at $\infty$ our variable $\lambda$ is rotated by a linear fractional transformation which is different for each line through the origin. Hence $\lambda$ cannot be extended to $P F^{-1}(\infty)$, and, in general, we would be surprised if a bundle produced by the action of our loop group extended through $\infty$.

This helps to explain a feature of our problem which appeared mysterious from the beginning: what could the infinitesimal action on the instanton solutions possibly look like?

The instanton $(S U(2))$ solutions form a sequence of connected, finitedimensional manifolds with boundary, one for every Chern class (or instanton number). They are parametrized by giving the center, strength, and orientation in the vector bundle of each instanton. Since there are no global solutions on $S^{4}$ of SDYME except the instantons, it seemed the loop group had to have some natural action on those parameters. Since none suggested itself, we were puzzled.

The answer is that the finite elements of the group produce sources, hence do not take the manifolds of instantons to themselves. What about the tangent space to the group? Since having a trivial Birkhoff decomposition is an open property, how can elements of the tangent space to the loop group avoid giving vector fields on the manifolds of instantons? The answer is that the action of the Kac-Moody elements "blows up" at $\infty$.

A method exists to make this precise. K. Uhlenbeck has shown [7] that a solution of SDYME on $R^{4}$ extends to a solution on $S^{4}$ iff its curvature is square integrable. An explicit computation would in all probability show that this is practically never preserved by the Kac-Moody action, since such a computation would include terms which do not even go to zero as $R \rightarrow \infty$.

The computation of the 1-parameter family of loop group actions provides an example of a Kac-Moody element which does not converge at $\infty$. Since as $\varepsilon \rightarrow 0$, the jumping lines converge to $\infty$, it is clearly impossible for the derivative of the family at $\varepsilon=0$ to converge at $\infty$.

We do not know how to explain why the element $g_{B}$ of the larger group described above takes the trivial bundle to a solution of SDYME which extends across $\infty$, or how to generalize the phenomenon.

Acknowledgements. The author wishes to thank Professor K. Uhlenbeck, under whose supervision this paper was written as a thesis at the University of Chicago, Mathematics Department. Without her gentle insistence, it would undoubtedly have made a very delayed appearance. I also wish to thank the Max Planck Institute in Bonn for their hospitality while this paper was partially written. I wish to thank L. Fania, G. Mülich, R. Narasimhan, M. Rothenberg, A. Soyring and H. Spindler for their helpful discussions. 


\section{References}

1. Adler, M., van Moerbeke, P.: Kowalewski's asymptotic method, Kac-Moody Lie algebras and regularization. Commun. Math. Phys. 83, 83-106 (1982).

2. Freund, P. G. O.: Superstrings from 26 dimensions. Institute for Theoretical Physics, University of California at Santa Barbara Preprint NSF-1TP-84-157.

3. (a) Chau, L. L., Ge, M. L., Wu, Y. S.: Kac-Moody algebra in the self dual Yang-Mills equation. Phys. Rev. D 25, 1086 (1982);

(b) Chau, L. L., Wu, Y. S.: More about hidden-symmetry algebra for the self-dual Yang-Mills system. Phys. Rev. D 26, 3581 (1982);

(c) Chau, L. L., Ge, M. L., Sinha, A., Wu, Y. S.: Hidden symmetry algebra for the self-dual YangMills equation. Phys. Let. 121 B, 391 (1983)

4. Dolan, L.: A new symmetric group of real self dual Yang-Mills theory. Phys. Lett. 113 B, 387-390 (1982)

5. Atiyah, M.: Geometry of Yang-Mills fields. Accademia Nazionale Dei Lincei Suola Normale Superiore Pisa 1979

6. Atiyah, M. F., Ward, R. S.: Instantons and algebraic geometry. Commun. Math. Phys. 55, 117-124 (1977)

7. Uhlenbeck, K.: Removable singularities in Yang-Mills fields. Bull. Am. Math. Soc. 1, 579-581 (1979)

8. Atiyah, M., Hitchin, N., Singer, I.: Self-duality in four dimensional Riemannian geometry. Proc. R. Soc. Lond. A 362, 420-461 (1978)

9. Wu, Y. S., Ge, M. L.: A simplified derivation of the Geroch group in two-dimensional reduced gravity. J. Math. Phys. 24, 1187 (1982), and references listed therein

10. Dolan, L.: Kac-Moody algebra is hidden symmetry of Chiral models. Report \#RU81/B/5, the Rockefeller University, New York, NY 1981

11. Bohr, H., Ge, M. L., Volovich, T. V.: New hidden symmetries in 2-dimensional models. Report \#IC/84/165, International Centre for Theoretical Physics, Trieste, Italy 1984

12. Pohlmeyer, K.: Integrable hamiltonian systems and interactions through quadratic constraints. Commun. Math. Phys. 46, 207-221 (1976)

13. Ernst, F. J.: New formulation of the axially symmetric gravitational field problem. Phys. Rev. 167, 1175 (1968)

14. Xanthopoulos, B. C.: A geometric notion of complete integrability, forthcoming

15. Witten, L.: Static axially symmetric solutions of self-dual $S U(2)$ gauge fields in euclidean fourdimensional space. Phys. Rev. D19, 218 (1979)

16. Okonek, C., Spindler, H.: Vector bundles on complex projective spaces. Boston: Birkhauser 1980.

17. Birkhoff, G. D.: A theorem on matrices of analytic functions. Math. Ann. 74, 122 (1913)

18. Brieskorn, E.: Über holomorphe $P_{n}$-bündel über $P_{1}$. Math. Ann. 157, 343-357 (1965)

19. Ablowitz, M., Segur, H.: Solitons and the inverse scattering transform. Philadelphia: SIAM 1981

20. Yang, C. N.: Conditions of self-duality for $S U(2)$ gauge fields on euclidean four-dimensional space. Phys. Rev. Lett. 38, 1377 (1977)

21. Chau, L. L., Prasad, M. K., Sinha, A.: Some aspects of the linear system for self-dual Yang-Mills fields. Phys. Rev. D 24, 1574 (1981)

22. Soyring, A.: Private communication

23. Belavin, A. A., Zakharov, V. E.: Yang-Mills equation as inverse scattering problem. Phys. Lett. 23 B, 53 (1978)

24. Prasad, M. K., Sinha, A., Chau Wang, L. L.: Parametric Bäcklund transformations for self-dual $S U(N)$ Yang-Mills fields. Phys. Rev. Lett. 43, 750 (1979)

25. Zakharov, V. E., Shabat, A. B.: Integration of nonlinear equations of mathematical physics by the method of inverse scattering II. Funct. Anal. Appl. 13, 13 (1979)

26. Ueno, K., Nakamura, Y.: Transformation theory for anti self dual equation and the RiemannHilbert problem. Phys. Lett. 109 B, 273 (1982)

27. Wu, Y. S.: The group theoretical aspects of infinitesimal Riemann-Hilbert problems and hidden symmetries. Commun. Math. Phys. 90, 461-472 (1983)

28. Sorkin, R.: Kaluza-Klein monopole, Phys. Rev. Lett. 51, 87 (1983)

29. Gurses, M., Xanthopoulos B. C.: Axially symmetric, static self-dual $S U(3)$ gauge fields and stationary Einstein-Maxwell metrics. Phys. Rev. D 261912 (1982). 
30. Mazur, P. O.: Acta Phys. Polon. B 14219 (1983)

31. Forgacs, P., Horvath, Z., Palla, L.: Generating the Bogomolny-Prasad-Sommerfield one-monopole solution by a Bäcklund transformation. Phys. Rev. Lett. 45, 505 (1980).

Communicated by S.-T. Yan

Received October 22, 1985; in revised form October 13, 1986 